Supporting information for:

\title{
A Spectroscopically-Validated Computational Investigation of Viable Reaction Intermediates in the Catalytic Cycle of the Reductive Dehalogenase PceA
}

Elizabeth D. Greenhalgh ${ }^{\dagger}$, Cindy Kunze ${ }^{\ddagger}$, Torsten Schubert ${ }^{\ddagger}$, Gabriele Diekert ${ }^{\ddagger}$, Thomas C. Brunold $^{\dagger, *}$

$\dagger$-Department of Chemistry, University of Wisconsin-Madison, Madison, Wisconsin 53706, United States

+-Department of Applied and Ecological Microbiology, Institute of Microbiology, Friedrich Schiller University, 07743 Jena, Germany

* E-mail: brunold@chem.wisc.edu. Phone: (608) 265-9056

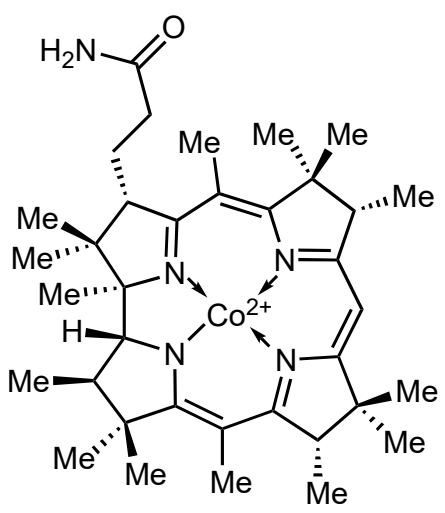

Figure S1. Truncation of $\mathrm{Cbl}$ model used for the QM region of QM/MM optimizations and for TD-DFT calculations. 


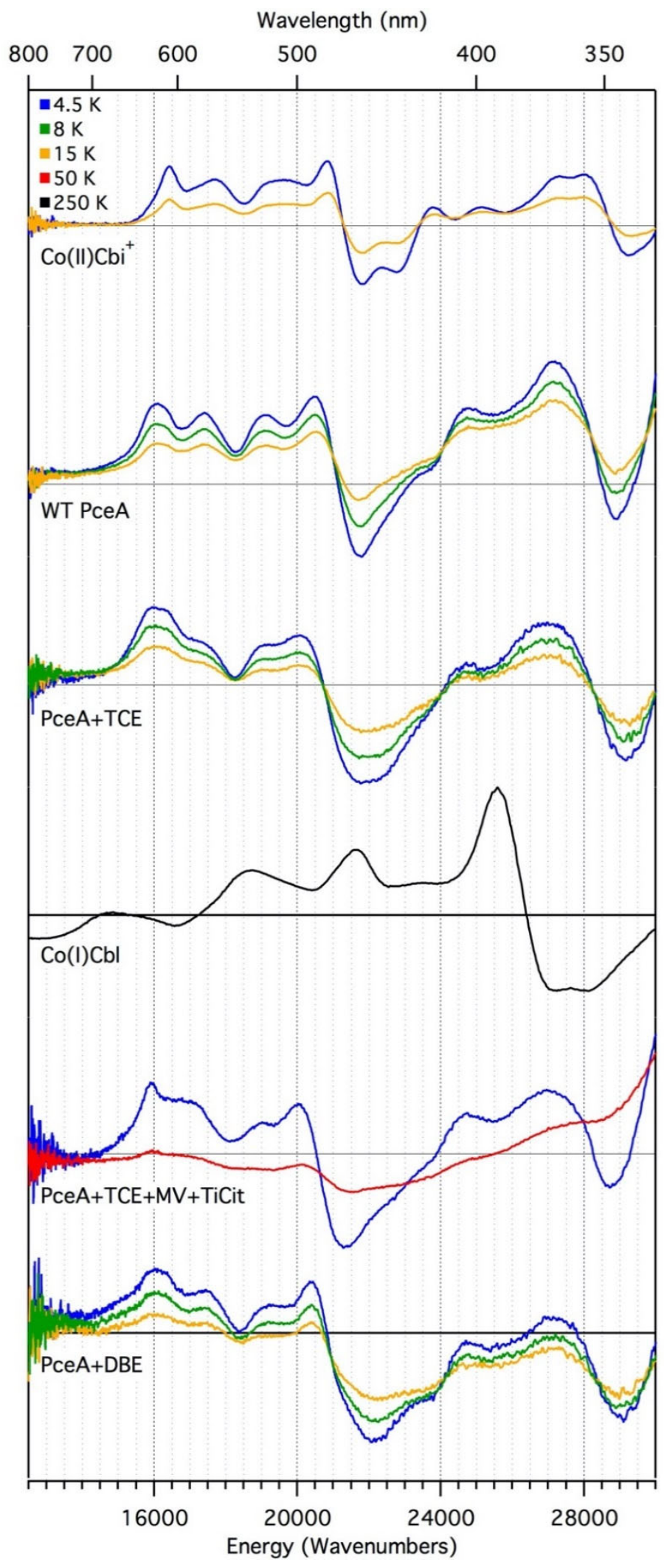

Figure S2. Temperature dependence of MCD spectra. Note that the MCD spectrum of Co(I)Cbl shows no temperature dependence because this species is diamagnetic. 
Table S1. Co Spin Densities for Spin Unrestricted QM/MM Models

\begin{tabular}{c|c} 
QM/MM Model & Mulliken Spin Density on Co \\
\hline \hline PceA & 1.0523 \\
\hline PceA + TCE & 1.0984 \\
\hline SET & 1.0981 \\
\hline Cl-Adduct, Homolytic cleavage & 1.0415
\end{tabular}

